\title{
Assessment of an ICU-specific, electronic medical summary tool against traditional dictation to reduce communication gaps during ICU-to-inpatient transitions-in-care
}

\author{
Mungunzul M. Amarbayan ${ }^{1}$, Liam Whalen-Browne ${ }^{1}$, Rebecca Brundin-Mather ${ }^{2}$, Devika \\ Kashyap $^{3}$, Khara Sauro ${ }^{1,4}$, Andrea Soo ${ }^{1,3}$, Jeanna Parsons Leigh ${ }^{1,4}$, Thomas Stelfox ${ }^{1,2,3,4}$ \\ ${ }^{1}$ Department of Critical Care Medicine, Cummings School of Medicine, University of Calgary \\ ${ }^{2}$ W21C Research and Innovation Centre, O'Brien Institute of Public Health, Cummings School of \\ Medicine, University of Calgary \\ ${ }^{3}$ Critical Care Medicine, Alberta Health Services \\ ${ }^{4}$ Department of Community Health Sciences \& O'Brien Institute of Public Health, Cummings \\ School of Medicine, University of Calgary
}

\begin{abstract}
Transition from the intensive care unit (ICU) to an inpatient unit is a vulnerable period where communication gaps between medical teams may be associated with preventable adverse events. The transition-in-care (TIC) summary encompasses essential clinical information and facilitates seamless continuity of patient care between sending and receiving healthcare teams. Yet, current dictation practices often produce summaries of suboptimal quality that result in delayed or incomplete information. An electronic TIC summary tool, an alternative method to dictation, standardizes information, which may ensure more timely and complete communication to reduce information breakdowns and delays. In order to standardize information communicated during ICU-to-inpatient transitions, an ICU-specific, electronic TIC summary tool was implemented in four adult ICUs in the Calgary zone. It is hypothesized that implementation of the electronic summary will improve completeness and timelines of TIC summaries. A multiple baseline study design was used to evaluate the implementation of the electronic TIC summary. ICUs continued dictation practices for a baseline period, until the electronic tool was implemented sequentially (in a randomized order) to each ICU and evaluated for six months following implementation. Post-implementation, providers had the option to dictate or use the electronic summary. The primary outcome was a binary measure of both completeness of four critical elements and availability of the TIC summary at ICU release. Preliminary results were obtained for two months of baseline $(n=48)$ and post-implementation $(n=48)$ from one ICU. Post-implementation summaries contained four critical elements and were more frequently available at ICU transfer than pre-implementation dictations (73\% versus $2 \%, p<0.001)$. More post implementation summaries contained completion of essential information (median $88 \%$ versus $63 \%, \mathrm{p}<0.001$ ) and had greater availability during transition $(90 \%$ versus $73 \%, p=0.04)$ than pre-implementation dictations. With data collection scheduled to end in June 2019, we anticipate full study results to be available fall 2019. Preliminary results post-implementation suggest greater completion and faster availability for the receiving clinicians. It is anticipated that full study findings will add to the current literature on the effect of computerized tools for reducing communication gaps between ICU and inpatient units during transitions-in-care to ultimately improve patient safety.
\end{abstract}

Cite as: Amarbayan M., Whalen-Browne L., Brundin-Mather R., Kashyap D., Sauro K. Soo A. Parsons Leigh J., Stelfox HT. 2019. Assessment of an ICU-specific, electronic medical summary tool against traditional dictation to reduce communication gaps during ICU-to-inpatient transitions-in-care. Alberta Academic Review, Vol 2 (3) 16, CASCH Special Issue (not peer-reviewed), DOI: 10.29173/aar82. 